\title{
INCENTIVES AND THE SEARCH FOR UNKNOWN RESOURCES SUCH AS WATER*
}

\author{
Jean-Jacques Laffont ${ }^{\dagger}$
}

January 27, 2003

*This paper was prepared for the talks given at the University of California at Berkeley and Davis and sponsored by the Giannini Foundation and for the book Frontiers in Water Resource Economics.

†Université Toulouse I, University of Southern California and CNRS. 


\section{Introduction}

The delegation of discovery tasks is quite common. Researchers are funded by public and private organizations for discovering new theorems, new computer algorithms, new engineering processes. Actually most of the $\mathrm{R} \& \mathrm{D}$ efforts are delegated by principals to agents through labor contracts. Multinational companies are often delegated the search and exploitation of oil fields or other natural resources such as water. Communities often own in common resources of unknown magnitudes like, water, wood, plants, fruits, game in forests or fish in rivers and oceans. In general, communities specialize some individuals (hunters, fishermen) to look for these resources.

The R\&D literature has well taken into account the randomness of discoveries and the need to structure contracts for giving proper incentives or effort to the agents in charge of R\&D tasks. One essential feature of the discovery process which has not been taken into account is that, almost by definition, the nature or size of the discovery is private information of the agent who makes the discovery. This may lead to opportunistic behavior of agents.

Taking advantage of the "no slavery" conditions of labor contracts researchers may hide their true discovery, renege on their labor contract and exploit their discovery outside the principal-agent relationship. Companies which are delegated by countries the search for natural resources may take advantage of the lack of technical expertise of these countries to hide some of their discoveries or even flee the country with their discovery. Similarly, hunters or fishermen may hide some of their findings and consume them secretly.

The common structure of these examples is a principal-agent problem in which a principal (the company, the country, the village) delegates to an agent the search for a resource of unknown size, and the outcome of the search is partially nonverifiable, and contracts are imperfect. In this paper we study this contractual problem by modeling the imperfection of contracts as an imperfection in the enforcement of contracts combined with limited liability constraints, noticeable characteristics of LDCs.

This paper is related to several strands of the economics literature. The first one is principal-agent models with interim status-quo payoffs which are state dependent (Lewis and Sappington (1989), Laffont and Tirole (1990), Maggi and Rodriguez-Clare (1995), Jullien (2000)). In our analysis the imperfection of enforcement will yield such interim constraints despite the fact that contracts are signed ex ante. The second one is the law and economics literature initiated by Becker (1968), Stigler (1970), Becker and Stigler (1974) about the imperfect enforcement of laws, rules and contracts. Even though it would be desirable to derive this imperfection from explicit transaction costs of the enforcement mechanism we will adopt a rather ad hoc formulation as in this literature. The third 
strand of the related literature concerns the manipulation of endowments in mechanism design (Postlewaite (1979), Hurwicz, Maskin and Postlewaite (1982), Green and Laffont (1986)). However, for reasons to be given below we will not use the major insight of these papers which is to argue that one can only lie downward about endowments.

Section 2 sets up the model and derives the optimal contract under complete information. Section 3 derives the precise structure of the optimal contract under asymmetric information when enforcement is imperfect. It entails efficient sharing of the resource between the principal and the agent or distortions due to countervailing incentives. These characteristics imply in Section 4 that the principal has the perverse incentive to discour-

age the agent's effort for high quality discovery. Section 5 shows how an improvement of enforcement may restore the optimality of contract and reverse these perverse incentives. Various extensions are discussed in Section 6. It is shown in particular that the details of enforcement imperfections matter a lot for the qualitative features of the optimal contract. Concluding comments are gathered in Section 7.

\section{The Model}

We consider a principal-agent relationship in which a principal delegates to an agent the search for a resource of unknown magnitude. For reference we consider in this section the case where the amount $\theta$ of resource is known.

The principal's utility function is $u(q)-t$, with $u^{\prime}>0, u^{\prime \prime}<0$, and where $q$ is the quantity of the resource obtained by the agent, while $t$ is the monetary payment made by the principal to the agent.

The agent's utility function is $u(\theta-q)+t$.

Under complete information the principal maximizes his utility under the participation constraint of the agent

$$
u(\theta-q)+t \geq 0
$$

For each $\theta$ the optimal solution is characterized by equal sharing of the good with $t=-u\left(\frac{\theta}{2}\right)$.

\section{Optimal Contract Under Asymmetric Information}

The amount discovered $\theta$ is now private information of the agent. $\theta$ can take two values $\underline{\theta}$ and $\bar{\theta}$ with respective probabilities $1-\nu$ and $\nu$. From the revelation principle we know that we can restrict the analysis to incentive compatible menus of contracts $\{\underline{t}, \underline{q} ; \bar{t}, \bar{q}\}$. 
Incentive compatibility is characterized by the following two incentive compatibility constraints

$$
\begin{aligned}
& u(\bar{\theta}-\bar{q})+\bar{t} \geq u(\bar{\theta}-q)+\underline{t} \\
& u(\underline{\theta}-\underline{q})+\underline{t} \geq u(\underline{\theta}-\bar{q})+\bar{t} .
\end{aligned}
$$

The contract between the principal and the agent is signed at the ex ante stage and subject to the agent's ex ante participation constraint:

$$
\nu(u(\bar{\theta}-\bar{q})+\bar{t})+(1-\nu)(u(\underline{\theta}-\underline{q})+\underline{t}) \geq 0 .
$$

Furthermore, we assume that they are some enforcement difficulties which depend on the legal environment. More specifically, ${ }^{3}$ we suppose that the agent can renege ${ }^{4}$ on the contract and disappear with the amount $\Delta \theta$ of the resource if he discovers $\bar{\theta}$ and with zero resource if he discovers $\underline{\theta}$. This leads to the additional enforcement or renegation constraints:

$$
\begin{aligned}
\underline{U} & =u(\underline{\theta}-\underline{q})+\underline{t} \geq 0 \\
\bar{U} & =u(\bar{\theta}-\bar{q})+\bar{t} \geq u(\Delta \theta) .
\end{aligned}
$$

The principal's best menu of contracts maximizes, under (1) to (4) the following objective function: ${ }^{5}$

$$
\nu(u(\bar{q})-\bar{t})+(1-\nu)(u(\underline{q})-\underline{t}) .
$$

In the absence of enforcement constraints, the principal facing a risk neutral agent with an ex ante participation constraint would achieve his first-best. ${ }^{6}$ Note also that (3) is implied by (4) and (5), so that the only constraints to consider are (1) (2) (4) (5). The problem becomes then similar to a principal agent problem with interim participation constraints and type-dependent status-quo utility levels for the agent. ${ }^{7}$ We have potentially 5 regimes of binding constraints, but the concavity of $u(\cdot)$ implies that (5) is always binding, so that we need only to consider three regimes. Intuitively, the righthand side of the enforcement constraint (5) is always higher than the information rent $R=u(\bar{\theta}-\underline{q})-u(\underline{\theta}-\underline{q})$ required from the incentive constraints (see Appendix 1).

\footnotetext{
${ }^{3}$ Alternative specifications of the enforcement constraints will be discussed in Section 6 .

${ }^{4}$ The principal could avoid this ex post opportunistic behavior by requiring a bond which would be lost if reneging occurred. However it is assumed that the lack of wealth of the agent makes this strategy impossible.

${ }^{5}$ We assume parameter values such that it is never in the principal's interest to opt for the shutdown of type $\underline{\theta}$ or for giving up the enforcement of type $\bar{\theta}$ 's contract.

${ }^{6}$ See for example Laffont and Martimort (2002), Chapter 2.

${ }^{7}$ See Lewis and Sappington (1989), Maggi and Rodriguez (1995), Jullien (2000), and Laffont and Martimort (2002) Chapter 3 for a simple exposition for 2 types.
} 
Regime 1: This regime obtains when only the enforcement constraints are binding. Substituting these constraints in the principal's objective function and maximizing with respect to $\underline{q}, \bar{q}$, we obtain efficient sharing. However, this regime is valid only if the $\underline{\theta}-$ agent's incentive constraint is satisfied, which amounts to $\Delta \theta>\underline{\theta}$. In this case, the principal's expected welfare is

$$
W_{1}=2 \nu u\left(\frac{\bar{\theta}}{2}\right)+2(1-\nu) u\left(\frac{\underline{\theta}}{2}\right)-\nu u(\Delta \theta) .
$$

With respect to the first best, the principal loses only $\nu u(\Delta \theta)$.

In the other two regimes we have countervailing incentives, i.e., it is the incentive constraint of the "bad" type $\underline{\theta}$ which is binding.

Regime 3: The enforcement constraint of the good type $\bar{\theta}$ and the incentive constraint of the bad type $\underline{\theta}$ are binding. Optimizing quantities we get:

$$
\begin{aligned}
\underline{q}_{3} & =\underline{q}^{*}=\frac{\underline{\theta}}{2} \\
u^{\prime}\left(\bar{q}_{3}\right) & =u^{\prime}\left(\bar{\theta}-\bar{q}_{3}\right)-\frac{(1-\nu)}{\nu}\left(u^{\prime}\left(\underline{\theta}-\bar{q}_{3}\right)-u^{\prime}\left(\bar{\theta}-\bar{q}_{3}\right)\right) .
\end{aligned}
$$

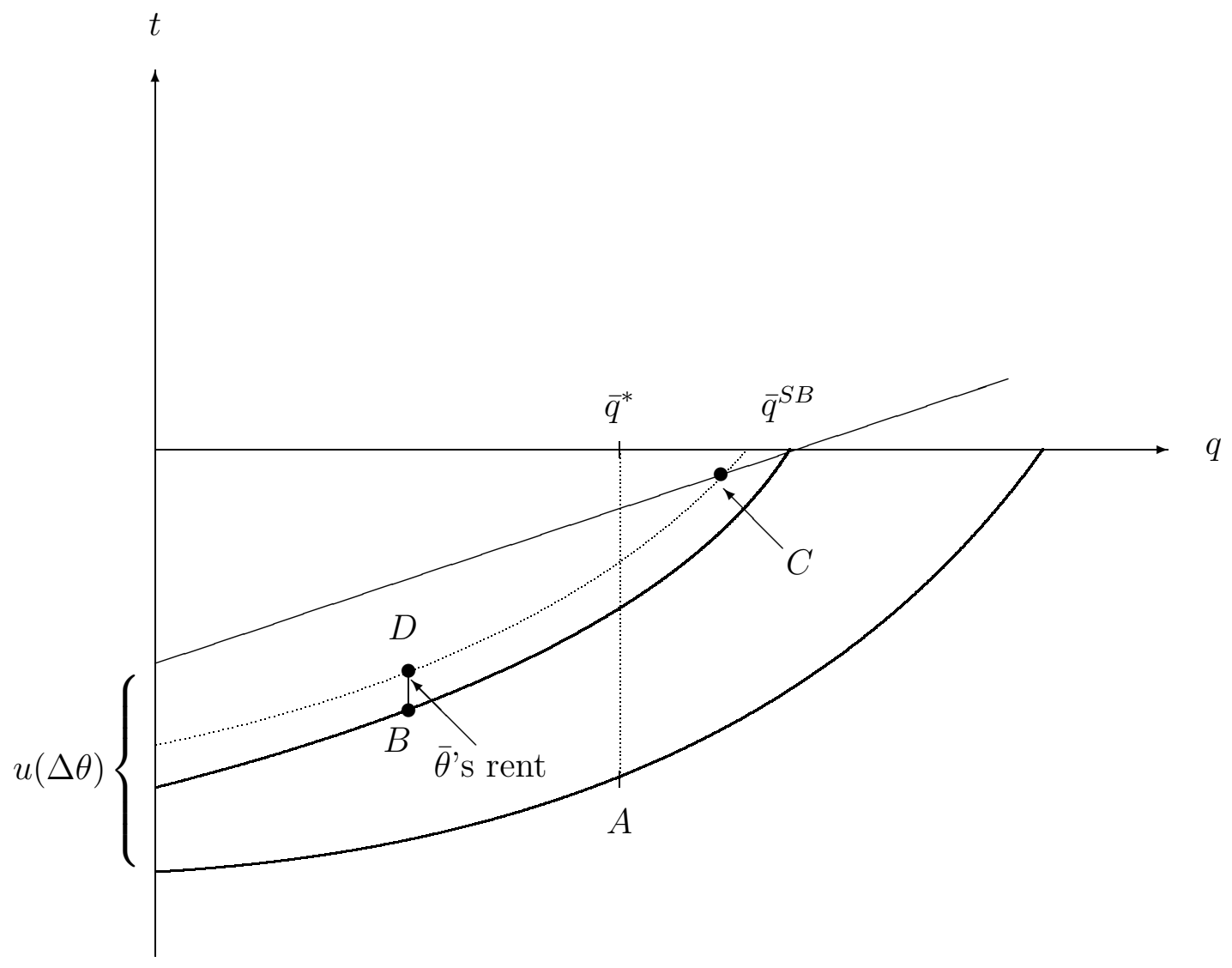

Figure 1: Optimal Contract in Regime 2. 
The optimal pair of contracts $(C, D)$ entails an upward distortion to $\bar{q}_{2}=\bar{q}^{S B}$, and in addition provides a rent to type $\underline{\theta}$.

Since $u(\cdot)$ is concave, (8) entails an upward distortion (see Figure 1 ) of $\bar{q}$ in order to decrease the information rent of the $\underline{\theta}$-type, namely $u(\Delta \theta)-(u(\bar{\theta}-\bar{q})-u(\underline{\theta}-\bar{q}))$, which is decreasing in $\bar{q}$. The principal's expected welfare is then

$$
\begin{aligned}
W_{2}= & \nu\left(u\left(\bar{q}_{3}\right)+u\left(\bar{\theta}-\bar{q}_{3}\right)\right)-\nu u(\Delta \theta) \\
& +2(1-\nu) u\left(\frac{\underline{\theta}}{2}\right)-(1-\nu)(u(\Delta \theta)-(u(\bar{\theta}-\bar{q})-u(\underline{\theta}-\underline{q}))) .
\end{aligned}
$$

However, this case is valid only if the $\underline{\theta}$ agent's participation constraint is satisfied, i.e., if

$$
u(\Delta \theta)-\left(u\left(\bar{\theta}-\bar{q}_{3}\right)-u\left(\underline{\theta}-\bar{q}_{3}\right)\right) \geq 0
$$

i.e., if $\bar{q}_{3} \leq \underline{\theta}$.

If (9) is not satisfied, we have regime 2 which connects Regimes 1 and 3. In Regime $2, \bar{q}_{2}=\underline{\theta}$ and $\underline{q}_{2}$ still equates $\frac{\theta}{2}$ with both enforcement constraints binding and defining the transfers. Then, the principal's expected welfare is

$$
W_{2}=\nu u(\underline{\theta})+2(1-\nu) u\left(\frac{\underline{\theta}}{2}\right) \text {. }
$$

Summarizing we have:

Proposition 1 : The optimal menu of contracts entails:

i) Efficient sharing if the asymmetry of information is large enough $(\Delta \theta>\underline{\theta})$.

ii) Countervailing incentives and downward distortions of the quantity allocated to the $\bar{\theta}$-agent, otherwise.

For the example $u(x)=x-\frac{x^{2}}{2}$ we give in Figure 2 the typical profile of quantity allocated to the $\bar{\theta}$-agent. 


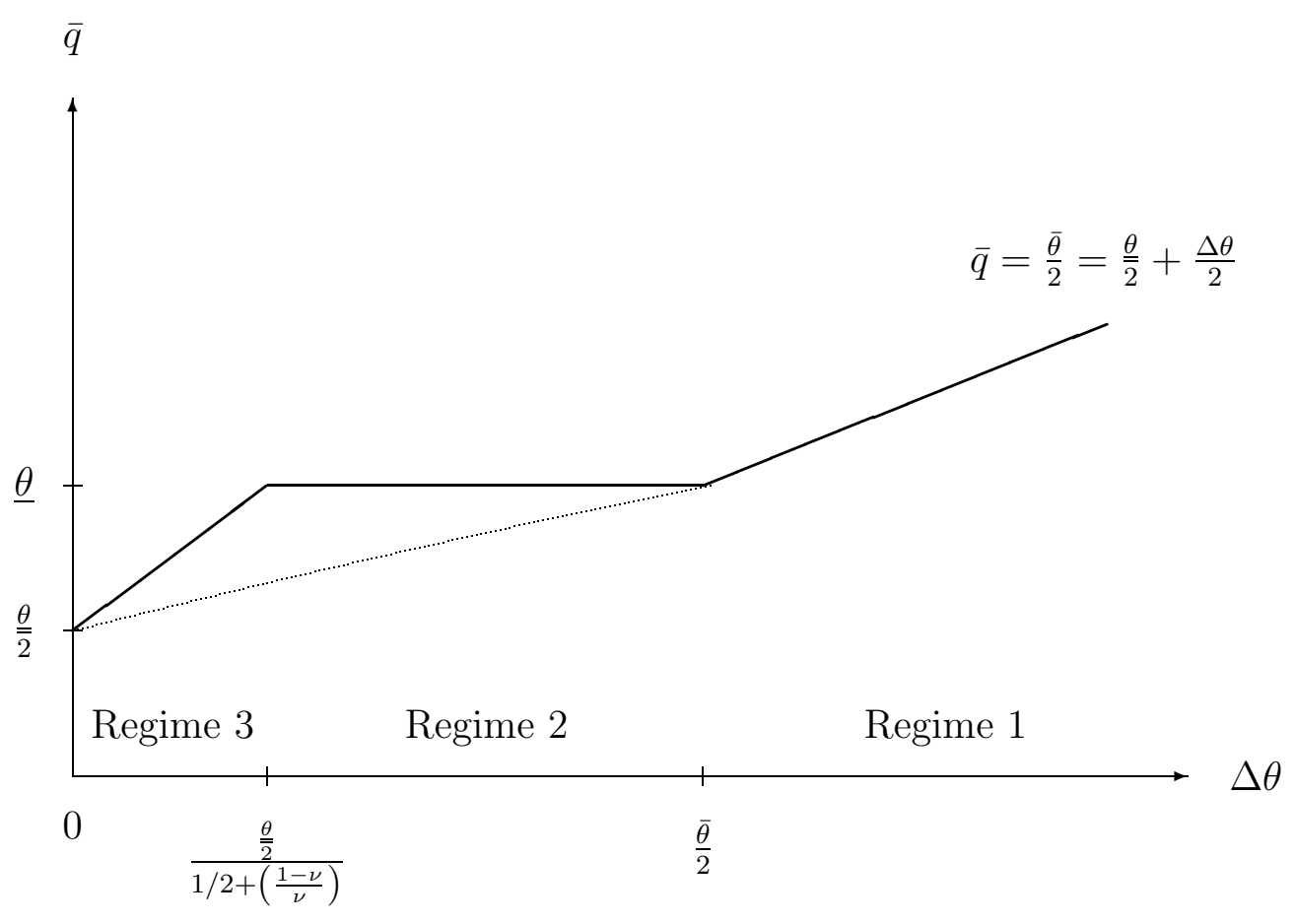

Figure 2

If $\nu>\frac{2}{3}$, Regime 3 does not exist.

So far, the main implication of enforcement constraints is that they may yield unexpected distortions of the optimal contract. Indeed, one might have expected intuitively that the agent should receive a higher share of the product when $\theta=\bar{\theta}$ in order to avoid untruthful behavior. It is on the contrary, an upward distortion of his share which is optimal. This is due to the fact that the strength of the enforcement constraint is so strong that in order to retain the $\bar{\theta}$-agent, the principal must offer him a contract so favorable that it becomes attractive to the $\underline{\theta}$-type. This calls for a distortion of the $\bar{\theta}$-contract aimed at avoiding this mimicking behavior.

In the next section, we see that these characteristics of the optimal contract have surprising effects on the principal's incentives to favor large discoveries.

\section{Incentives for Discovery Effort}

So far, the agent was unable to affect by his own behavior the probability distribution of the resource to discover. We assume now that by exerting an effort which costs him $\psi$ the agent increases the probability of a $\bar{\theta}$-discovery from $\nu_{0}$ to $\nu_{1}>\nu_{0}$ and let $\Delta \nu=\nu_{1}-\nu_{0}$. 
Consider ${ }^{8}$ Regime 1 . Observe that the welfare obtained by the principal in this regime

$$
W_{1}(\nu) \equiv 2\left(\nu u\left(\frac{\bar{\theta}}{2}\right)+(1-\nu) u\left(\frac{\hat{\theta}}{2}\right)\right)-\nu u(\Delta \theta),
$$

is decreasing in $\nu$, and that the definition of Regime $1(\Delta \theta>\underline{\theta})$ is independent of $\nu$. So, the principal wishes to discourage the agent from making an effort. For this purpose, he solves the following program

$$
\begin{gathered}
\max _{(\bar{q}, \bar{t} ; \underline{q}, \underline{t})} \nu_{0}(u(\bar{q})-\bar{t})+\left(1-\nu_{0}\right)(u(\underline{q})-\underline{t}) \\
u(\underline{\theta}-\underline{q})+\underline{t} \geq 0 \\
u(\bar{\theta}-\bar{q})+\bar{t} \geq u(\Delta \theta) \\
\nu_{0}(u(\bar{\theta}-\bar{q})+\bar{t})+\left(1-\nu_{0}\right)(u(\underline{\theta}-\underline{q})+\underline{t}) \\
\geq \nu_{1}(u(\bar{\theta}-\bar{q})+\bar{t})+\left(1-\nu_{1}\right)(u(\underline{\theta}-\underline{q})+\underline{t})+\psi .
\end{gathered}
$$

With (10) binding, the moral hazard incentive constraint (12) becomes

$$
u(\bar{\theta}-\bar{q})+\bar{t} \geq \frac{\psi}{\Delta \nu}
$$

so that (11) (12) become

$$
u(\bar{\theta}-\bar{q})+\bar{t} \geq \max \left(u(\Delta \theta), \frac{\psi}{\Delta \nu}\right) .
$$

If $u(\Delta \theta)<\frac{\psi}{\Delta \nu}$, the principal succeeds in discouraging the agent from making an effort with the efficient sharing of resources and transfers defined by (10) and (11) binding. Then, the principal obtains the expected welfare $W_{1}\left(\nu_{0}\right)$.

For $\Delta \theta$ large, the principal gives up discouraging the agent from exerting effort and obtains the expected welfare $W_{1}\left(\nu_{1}\right)$.

For intermediary values of $\Delta \theta$ but larger than $\Delta \theta_{0}=u^{-1}(\psi / \Delta \nu)$, the principal relaxes the participation constraint of the $\underline{\theta}$-type to still discourage effort, i.e., in such a way that (noting that (11) remains binding)

$$
\left(\nu_{1}-\nu_{0}\right)(u(\underline{\theta}-\underline{q})+\underline{t}-u(\Delta \theta))+\psi=0
$$

or

$$
u(\underline{\theta}-\underline{q})+\underline{t}=u(\Delta \theta)-\frac{\psi}{\Delta \nu} .
$$

\footnotetext{
${ }^{8}$ In Appendix 2, we show that the qualitative results obtained below hold also in Regimes 2 and 3.
} 
Efficient sharing still obtains and the principal' expected utility

$$
\tilde{W}_{1}\left(\nu_{0}\right)=W_{1}\left(\nu_{0}\right)-\left(1-\nu_{0}\right)\left(u(\Delta \theta)-\frac{\psi}{\Delta \nu}\right)
$$

Effort is not discouraged for $\Delta \theta \geq \Delta \theta_{1}$ such that

$$
W_{1}\left(\nu_{1}\right)=W_{1}\left(\nu_{0}\right)-\left(1-\nu_{0}\right)\left(u\left(\Delta \theta_{0}\right)-\frac{\psi}{\Delta \nu}\right) .
$$

We summarize these results and those obtained in Appendix 2 (see also Figure 3) by the following proposition.

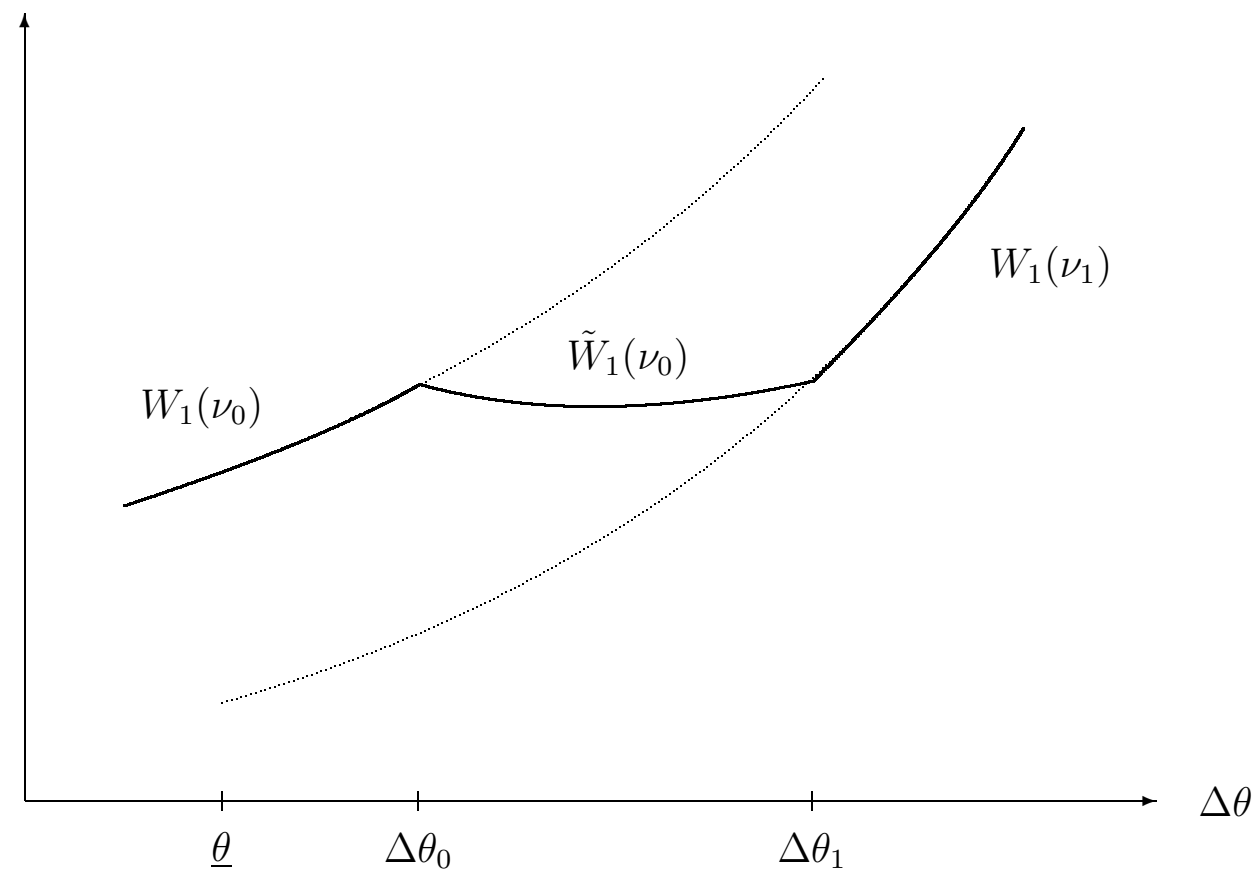

Figure 3

Proposition 2 : The principal will structure incentives in order to discourage effort. This is done without cost for small $\Delta \theta$. For large $\Delta \theta$ he gives up discouraging effort. For intermediary values of $\Delta \theta$ discouraging effort is costly and requires rewarding more the $\underline{\theta}$-agent..$^{9}$

Note that in the intermediary regime $\tilde{W}_{1}\left(\nu_{0}\right)$ is decreasing ${ }^{10}$ for $\Delta \theta$ close to $\Delta \theta_{0}$.

The striking consequence of the enforcement constraint is that the principal has the incentive to discourage effort. If possible he will even make difficult for the agent to

\footnotetext{
${ }^{9}$ If $u(\underline{\theta})<\frac{\psi}{\Delta \nu}$, Figure 5 is truncated.

$10 \frac{d \tilde{W}_{1}}{d \Delta \theta}=\nu_{0} u^{\prime}\left(\frac{\bar{\theta}}{2}\right)-u^{\prime}(\Delta \theta)<0$ at $\Delta \theta=\Delta \theta_{0}$, but ambiguous near $\Delta \theta_{1}$.
} 
increase his probability of a high discovery, but denying for example proper equipment. He will also structure incentive payments so that effort is discouraged, unless it is too costly. Not only is weak enforcement calling for distortions in general and is costly to the principal. It can set up very wrong incentives for progress in society.

The intuition for this result is simply that the principal benefits more from a $\underline{\theta}$-agent than from a $\bar{\theta}$-agent because of the cost of the enforcement constraint. Indeed

$$
\begin{aligned}
\underline{V} & =u(\underline{q})+\underline{t}=2 u\left(\frac{\underline{\theta}}{2}\right) \\
\bar{V} & =u(\bar{q})+\bar{t}=2 u\left(\frac{\bar{\theta}}{2}\right)-u(\Delta \theta)
\end{aligned}
$$

and $\bar{V}<\underline{V}$ since

$$
u\left(\frac{\bar{\theta}}{2}\right)-u\left(\frac{\theta}{2}\right)<u\left(\frac{\Delta \theta}{2}\right)<\frac{1}{2} u(\Delta \theta),
$$

from the concavity of $u(\cdot)$.

So as

$$
W=\nu \bar{V}+(1-\nu) \underline{V}, \quad \frac{\partial W}{\partial \nu}=\bar{V}-\underline{V}<0 .
$$

Nevertheless, note that $W_{1}(\nu)$ is increasing in $\Delta \theta$ as

$$
\frac{\partial W_{1}}{\partial \Delta \theta}=\nu\left(u^{\prime}\left(\frac{\bar{\theta}}{2}\right)-u^{\prime}(\Delta \theta)\right)>0 .
$$

Note that when we have countervailing incentives the principal's welfare is non increasing in $\Delta \theta$ and even decreasing in Regime 3. Then, the principal has even incentives to damage the quality of the high discovery.

In the next section we show how an improvement of enforcement may take the relationship out of the vicious circle emphasized above.

\section{$5 \quad$ Endogenous Enforcement}

Suppose now that with an ex ante expense of $c(p)$ the principal can improve enforcement. More specifically, with probability $p$ the agent is set at the zero utility level ${ }^{11}$ and with probability $1-p$ he escapes. Let us assume that $c(\cdot)$ is convex with the Inada conditions $c(0)=0, c^{\prime}(0)=0, \lim _{p \rightarrow 1} c^{\prime}(p)=\infty$.

The ex post enforcement constraint (5) becomes

$$
\bar{U}=u(\bar{\theta}-\bar{q})+\bar{t} \geq(1-p) u(\Delta \theta) .
$$

\footnotetext{
${ }^{11}$ Higher penalties are not possible because of limited liability constraints.
} 
The principal's problem can be rewritten

$$
\max _{\{\bar{q}, \bar{t} ; \underline{q}, \underline{t} ; p\}} \nu(u(\bar{q})-\bar{t})+(1-\nu)(u(\underline{q})-\underline{t})-c(p)
$$

s.t. (1) (2) (4) (13).

Thanks to enforcement expenditures it is now possible to have Regime 0, for which the $\bar{\theta}$-type incentive constraint and the $\underline{\theta}$-participation constraint are binding. Actually, it is never worth spending enforcement resources to make constraint (13) strict. So, in Regime 0, constraints (1) (3) (13) are binding. Substituting the transfers from (1) and (3) into the principal's objective function leads to:

$$
\max _{\{\bar{q}, \underline{q}, p\}} \nu(u(\bar{q})+u(\bar{\theta}-\bar{q})-(u(\bar{\theta}-\underline{q})-(\underline{\theta}-\underline{q})))+(1-\nu)(u(\underline{q})+u(\underline{\theta}-\underline{q}))-c(p)
$$

s.t.

$$
u(\bar{\theta}-\underline{q})-u(\underline{\theta}-\underline{q})-(1-p) u(\Delta \theta)=0 .
$$

The optimal solution is:

$$
\begin{aligned}
\bar{q}_{0} & =\bar{\theta} \\
u^{\prime}\left(\underline{q}_{0}\right) & =u^{\prime}\left(\underline{\theta}-\underline{q}_{0}\right)-\frac{\left(\nu-\frac{c^{\prime}\left(p_{0}\right)}{u(\Delta \theta)}\right)}{1-\nu}\left(u^{\prime}\left(\bar{\theta}-\underline{q}_{0}\right)-u^{\prime}\left(\underline{\theta}-\underline{q}_{0}\right)\right) \\
u\left(\bar{\theta}-\underline{q}_{0}\right)-u\left(\underline{\theta}-\underline{q}_{0}\right) & =\left(1-p_{0}\right) u(\Delta \theta) .
\end{aligned}
$$

This solution holds as long as the $\underline{\theta}$-agent's incentive constraint is not binding (i.e., for $\bar{\theta}>2 \underline{\theta}$ ) and as long as $c^{\prime}\left(p_{0}\right)<\nu u(\Delta \theta)$. We observe that the enforcement cost leads to a smaller distortion in $\underline{q}_{0}$. Indeed, when $\underline{q}_{0}$ is decreased to decrease the $\bar{\theta}$-agent's information rent one must take into account the addition enforcement cost due to the higher $p_{0}$ needed to maintain (16).

When $\Delta \theta$ decreases and $p_{0}$ reaches $p_{1}$ defined by $c^{\prime}\left(p_{0}\right)=\nu u(\Delta \theta)$, the $\bar{\theta}$-agent's incentive constraint becomes slack and we obtain Regime 1 with efficient sharing $p=p_{1}$ and transfers $\underline{t}=-u\left(\frac{\theta}{\overline{2}}\right), \bar{t}=-u\left(\frac{\bar{\theta}}{2}\right)+\left(1-p_{1}\right) u(\Delta \theta)$. When $\bar{\theta}<2 \underline{\theta}$, we have also two regimes. In Regime 3 , only the participation constraint of type $\bar{\theta}$ and the incentive constraint of type $\underline{\theta}$ are binding. We obtain immediately

$$
\begin{aligned}
\underline{q}_{3} & =\frac{\underline{\theta}}{2} \\
u^{\prime}\left(\bar{q}_{3}\right) & =u^{\prime}\left(\bar{\theta}-\bar{q}_{3}\right)-\frac{(1-\nu)}{\nu}\left(u^{\prime}\left(\underline{\theta}-\bar{q}_{3}\right)-u^{\prime}\left(\bar{\theta}-\bar{q}_{3}\right)\right) \\
c^{\prime}\left(p_{3}\right) & =u(\Delta \theta) .
\end{aligned}
$$


For $\Delta \theta$ higher $p$ is adjusted so that

$$
(1-p) u(\Delta \theta)=u(\bar{\theta}-\bar{q})-u(\underline{\theta}-\underline{q})
$$

and we obtain Regime 2 characterized by

$$
\begin{aligned}
\underline{q}_{2} & =\frac{\underline{\theta}}{2} \\
u^{\prime}\left(\bar{q}_{2}\right) & =u^{\prime}\left(\bar{\theta}-\bar{q}_{2}\right)+\frac{\left(1-\nu-\frac{c^{\prime}\left(p_{2}\right)-u(\Delta \theta)}{u(\Delta \theta)}\right)}{\nu}\left(u^{\prime}\left(\bar{\theta}-\bar{q}_{2}\right)-u^{\prime}\left(\underline{\theta}-\bar{q}_{2}\right)\right) \\
\left(1-p_{2}\right) u(\Delta \theta) & =u\left(\bar{\theta}-\bar{q}_{2}\right)-u\left(\underline{\theta}-\underline{q}_{2}\right) .
\end{aligned}
$$

Putting together these results we describe in Figure 4 the profile of optimal enforcement levels.

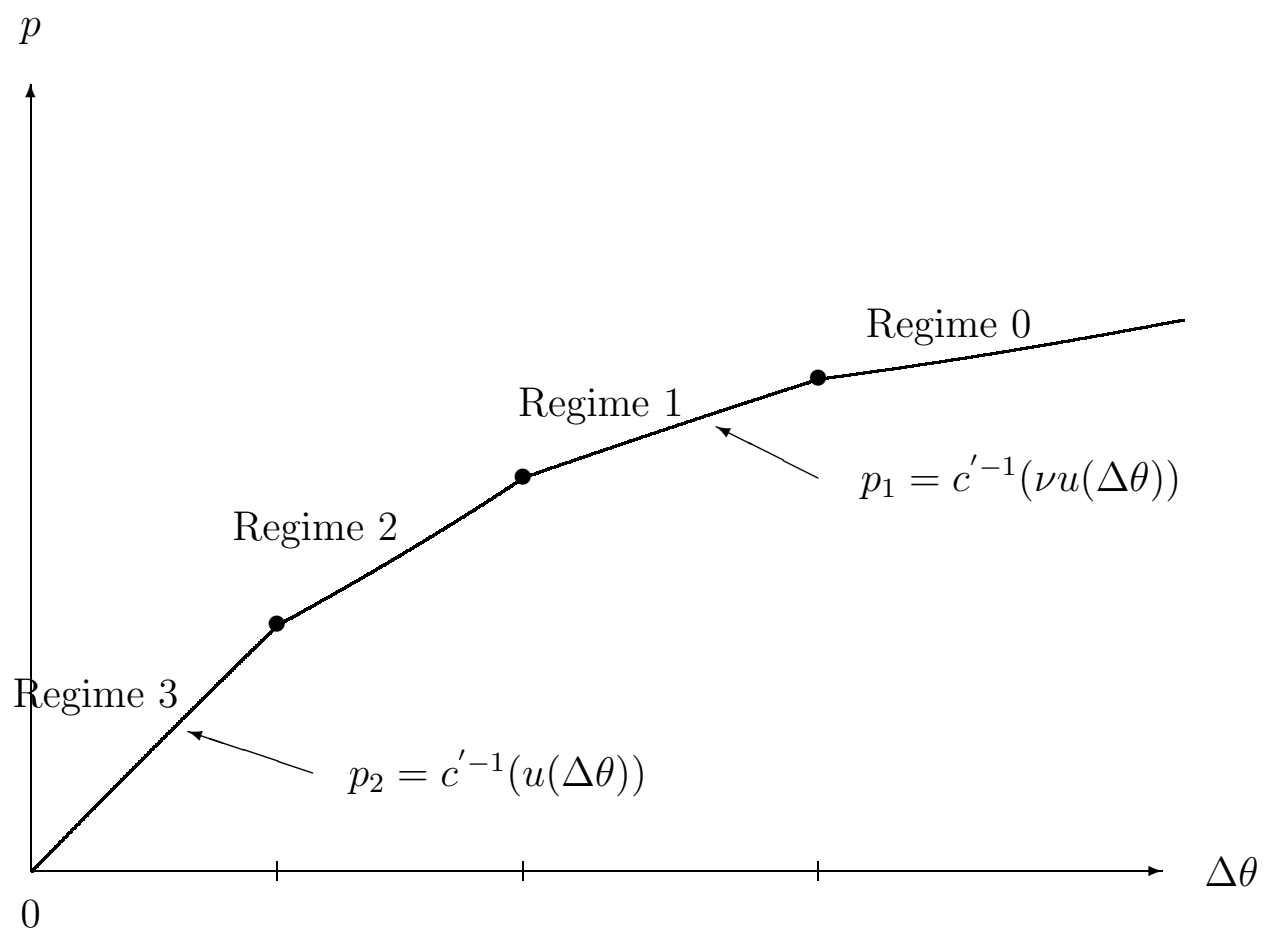

Figure 4

For the quadratic example we obtain in Figure 5 the quantity profiles. 


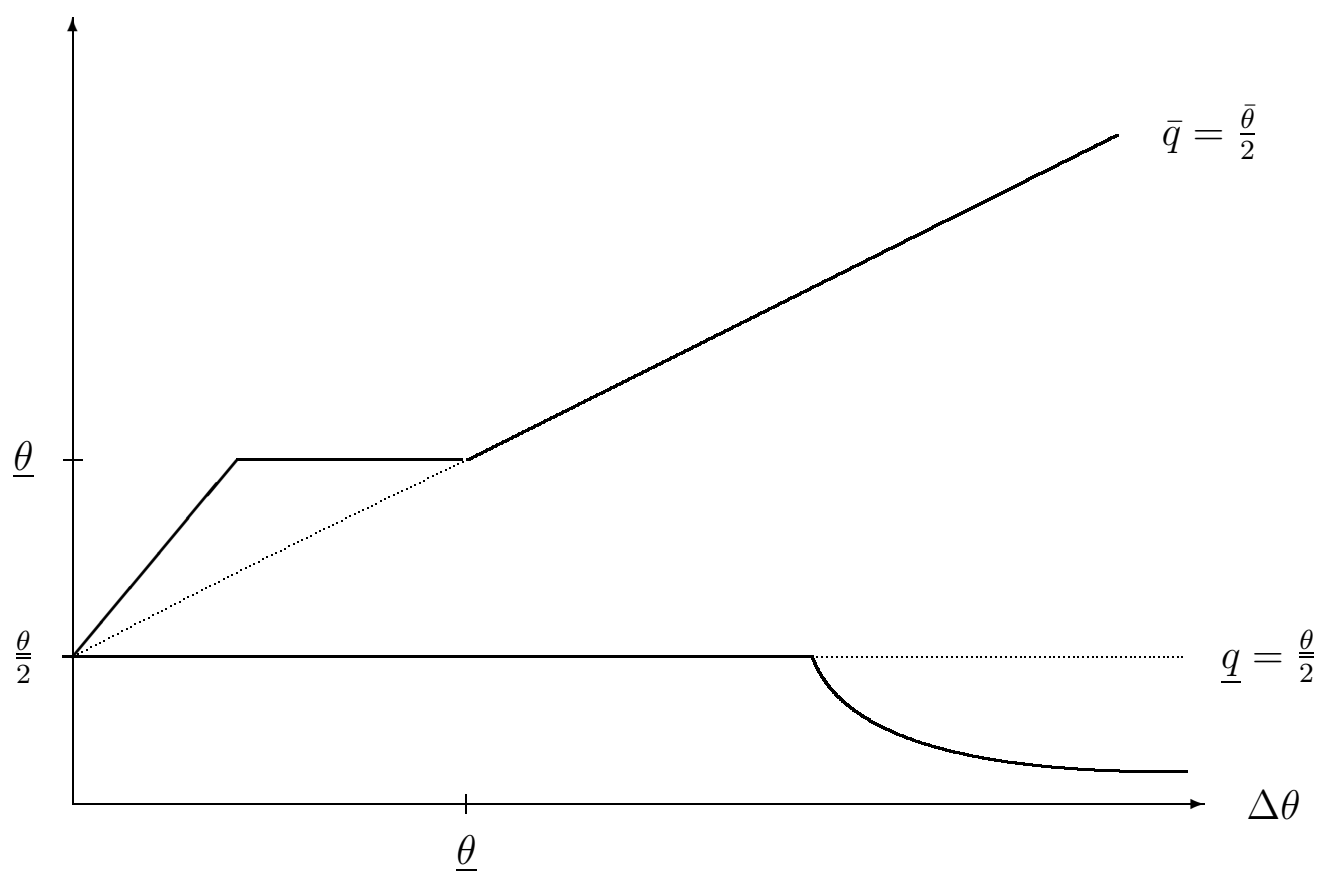

Figure 5

When better enforcement produces Regime 0 , the principal's welfare becomes increasing in $\nu$ and the principal has now incentives to induce effort. For a given level of $\psi$ they may not want to pay the price for it, but as $\Delta \theta$ increases they will indeed structure the incentives to induce effort (see Appendix 3).

Summarizing we have:

Proposition 3: When the cost of better enforcement is low enough the principal becomes interested in increasing $\nu$ for $\Delta \theta$ large enough. Then, he will structure incentives to induce effort. The domain of parameters where this phenomenon occurs expands as the quality of enforcement increases.

Note that better enforcement and lower powered incentives for production are complement instruments. Indeed, better enforcement creates higher incentives for discovery effort. This in turn creates a higher probability of having a $\bar{\theta}$-agent, therefore a higher desire to limit his information rent through a lower powered incentives for production. 


\section{$6 \quad$ Extensions}

\subsection{Sharing without Transfers}

In the absence of transfers, incentive constraints are much more constraining. A revelation mechanism is then simply a pair $(\underline{q}, \bar{q})$ of quantities provided to the principal.

The agent's incentive constraints then write:

$$
\begin{aligned}
& u(\bar{\theta}-\bar{q}) \geq u(\bar{\theta}-q) \\
& u(\underline{\theta}-\underline{q}) \geq u(\underline{\theta}-\bar{q}),
\end{aligned}
$$

implying $\underline{q}=\bar{q}=q$.

The principal's program reduces to

$$
\max _{q} u(q)
$$

s.t.

$$
\begin{aligned}
& u(\bar{\theta}-q) \geq u(\Delta \theta) \\
& u(\underline{\theta}-q) \geq 0,
\end{aligned}
$$

with the obvious solution $q=\underline{\theta}$. The agent keeps the surplus $\Delta \theta$ when it occurs.

Suppose that a contract (without transfers) can be signed ex ante. Then, the principal's program is constrained only by the agent's ex ante participation constraint

$$
\nu u(\bar{\theta}-q)+(1-\nu) u(\underline{\theta}-q) \geq 0,
$$

and the constraint $q \leq \underline{\theta}$. Again the optimal $q$ is $\underline{\theta}$. So, in the absence of transfers we obtain a very inefficient allocation of resources. ${ }^{12}$

\subsection{Partially Verifiable Resources}

One could argue that the agent can hide some of his discovery, but cannot claim he has discovered more than he has done in reality, because he must be able to show it. In other words, he can lie downward but not upward. ${ }^{13}$

\footnotetext{
${ }^{12}$ Of course, repeated relationships as one can find in villages of LDCs relax the incentive constraints through reputation effects.

${ }^{13}$ When the message space available to an agent depends on his type, the revelation principle may not hold (Green and Laffont (1986)). However, our simple case satisfies the necessary and sufficient condition obtained in Green and Laffont (1986) for the revelation principle to hold (see also Bull and Watson (2001)). The literature on manipulation of endowments (Postlewaite (1979), Hurwicz, Maskin and Postlewaite (1982)) makes a crucial use of the inability to lie upward.
} 
In this case, there is no incentive constraint for the $\underline{\theta}$-type, and the only binding constraints are the participation constraints with efficient sharing of the discovered resource.

However, there are cases where such a strategy of asking the agent to exhibit his discovery is not possible. If it is a discovery like a computer program, just showing it provides the resource to the principal. If it is a farmer upstream on a river the water he let flow downstream cannot be recaptured. Also the agent may have hidden resources which enable him to mimic the behavior of the $\bar{\theta}$-type. The model we have studied is designed to fit those situations.

\subsection{Modeling Imperfect Enforcement}

It turns out that the details of imperfect enforcement play a crucial role in determining the structure of the optimal contract and its impact on the incentives for discovery effort.

Let us denote more generally $w(\theta)$ the outside opportunity of type $\theta$ when he reneges on the contract. If we still normalize by $w(\underline{\theta})=0$ we have the "normal" case of Regime 0 if

$$
u\left(\bar{\theta}-\underline{q}^{S B}\right)-u\left(\underline{\theta}-\underline{q}^{S B}\right)>w(\bar{\theta})
$$

where $\underline{q}^{S B}$ is determined by

$$
u^{\prime}\left(\underline{q}^{S B}\right)=u^{\prime}\left(\underline{\theta}-\underline{q}^{S B}\right)-\frac{\nu}{1-\nu}\left(u^{\prime}\left(\bar{\theta}-\underline{q}^{S B}\right)-u^{\prime}\left(\underline{\theta}-\underline{q}^{S B}\right)\right) .
$$

We saw above that it is impossible if $w(\bar{\theta})=u(\bar{\theta}-\underline{\theta})$ and that it can become possible if $w(\bar{\theta})=(1-p) u(\bar{\theta}-\underline{\theta})$ for $p$ large enough.

An alternative formulation is

$$
w(\theta)=(1-p) u(\theta) \quad \text { for all } \theta
$$

Then, the rent of a good type

$$
u(\underline{\theta})+u\left(\bar{\theta}-\underline{q}^{S B}\right)-u\left(\underline{\theta}-\underline{q}^{S B}\right) \geq u(\bar{\theta}) \geq(1-p) u(\bar{\theta})
$$

since $u(\bar{\theta}-q)-u(\underline{\theta}-q)$ is increasing in $q$. We always have Regime 0 .

It is therefore important to understand how the imperfection of enforcement structures the outside opportunity levels of the reneging agents. We give below a few examples:

\section{i) Black market.}

Suppose that, when they carry out their contract, the principal and the agent can sell 
their share of the product on a idiosyncratic market each with the inverse demand function $P(\cdot)$ with $P(q) q$ concave.

Then

$$
\begin{aligned}
u(q) & =P(q) q \\
u(\theta-q) & =P(\theta-q)(\theta-q) .
\end{aligned}
$$

Alternatively the agent may renege the contract and sell on a black market with the inverse demand function $\tilde{P}(\cdot)$. This provides an alternative revenue of $\tilde{P}(\theta) \theta$ or $(1-p) \tilde{P}(\theta) \theta$ if the agent is caught with probability $p$.

Again, Regime 0 cannot hold if

$$
\tilde{P}(\underline{\theta}) \underline{\theta}+P\left(\bar{\theta}-\underline{q}^{S B}\right)\left(\bar{\theta}-\underline{q}^{S B}\right)-P\left(\underline{\theta}-\underline{q}^{S B}\right)\left(\underline{\theta}-\underline{q}^{S B}\right) \leq \tilde{P}(\bar{\theta}) \bar{\theta} .
$$

In words it means simply that the information rent

$$
\left(P\left(\bar{\theta}-\underline{q}^{S B}\right)\left(\bar{\theta}-\underline{q}^{S B}\right)-P\left(\underline{\theta}-\underline{q}^{S B}\right)\left(\underline{\theta}-\underline{q}^{S B}\right)\right)
$$

must be less than the difference of opportunities in the black market between the two types of agents. Clearly, all cases are possible depending on the demand function in the black market.

ii) Corruption of enforcer.

Suppose that leaving the country is only possible with the corruption of the customs officer; and suppose that a minimal bribe of $k$ in units of the good is needed to corrupt the officer and assume $\underline{\theta}<k$. Then

$$
\begin{aligned}
& w(\underline{\theta})=0 \\
& w(\bar{\theta})=u(\bar{\theta}-k) .
\end{aligned}
$$

This yields a model analogous to the one studied above. Clearly, the details of the corruption game are crucial. The agent might need the complicity of the officer to value the good and would have to share the good. Let $\alpha$ the share of the agent

$$
w(\theta)=u(\alpha \theta) .
$$

Then for $\alpha$ large enough, Regime 0 cannot hold while it does if $\alpha$ is small enough.

\section{iii) Joint Venture.}

Suppose that to value his discovery the agent must pay a fixed cost $k$, that his principal and maybe others have already sunk. 
Then $w(\theta)=u(\theta)-k$. The principal can implement Regime 0 and the more cheaply the larger is $k$. However, if other "principals" have also sunk the fixed cost, the agent may still renege and organize an auction between the principals. This would be the case of a researcher paid in his university to do research and who, after a large $\bar{\theta}$ discovery, would take advantage of the "no slavery" constraint in labor contracts to leave and offer his discovery to other universities. This illustrates the kind of inefficiency which may arise due to the "no slavery" condition. ${ }^{14}$

\subsection{Continuous Case}

The analysis can be easily extended to the case of continuous type $\theta$ in $[\underline{\theta}, \bar{\theta}]$. The objective function of the agent is then

$$
U(\theta)=u(\theta-q(\theta))+t(\theta)
$$

resulting in an incentive constraint (for Regime 0)

$$
\dot{U}(\theta)=u^{\prime}(\theta-q(\theta)) \text {. }
$$

It is then easy to see that Regime 0 is impossible if the slope of the information rent $u^{\prime}(\theta-q(\theta))$ is lower than the slope of the outside opportunity $w^{\prime}(\theta)$.

For example, if $w^{\prime}(\theta)=(1-p) u^{\prime}(\theta)$

$$
u^{\prime}(\theta-q(\theta))>(1-p) u^{\prime}(\theta)
$$

and Regime 0 always occur. If $w^{\prime}(\theta)=u^{\prime}(\theta-\underline{\theta})$ then for $\theta$ small,

$$
u^{\prime}(\theta-\bar{q}(\theta))<u^{\prime}(\theta-\underline{\theta})
$$

as $q(\theta)<\theta$ for $\theta$ in a neighborhood of $\underline{\theta}$. Then Regime 0 is impossible for $\theta$ close to $\underline{\theta}$. For large $\theta$ it becomes possible (see Appendix 4).

So what matters are not the absolute levels of information rents and outside opportunities, but their rate of growth in the parameter of asymmetric information.

\section{Conclusion}

We have studied a delegation problem in which the nonverifiability of the agent's discovery fuels the opportunistic behavior of agents who have high performances and may renege on

\footnotetext{
${ }^{14}$ As labor contracts do not allow for such bonds, firms or universities often circumvent partially this problem by complementing the salary with financial advantages (like mortgage loans) which result in high penalties if the employment relationship is broken.
} 
their contract and value their discovery outside the principal-agent relationship. A striking implication of the optimal contract is that it may destroy the incentive of the principal to provide good working conditions to the agent which would increase the probability of high discovery, and even favor the reward for low discovery to discourage agents to exert high levels of effort which would increase this probability of high discovery. Then, we have shown how an improvement of institutions in the form of better enforcement of contract, brought about by computer equipment for example, may reverse those perverse incentives. Finally, we have shown the need for a deeper analysis of the transaction costs of contract enforcement whose details affect considerably the structure of the optimal contract and the incentives it creates.

Beyond this main point, our analysis calls for further research in various directions. One is in labor economics and R\&D research where the traditional "no slavery" conditions which make easy for workers to end their employment relationship may have spectacular implications on incentives.

Another is the analysis of the impact of black markets on the structure of labor contracts in the formal economy. Also, it would be interesting to characterize the optimal auctions of contracts for discovery of resources when the nonverifiability and enforcement conditions of this paper hold. 


\section{Appendix 1}

The usual regime in models with adverse selection is to have the good type $\bar{\theta}$ 's participation constraint and the bad type $\underline{\theta}$ 's interim participation (here enforcement) constraint binding. Then

$$
\begin{aligned}
\bar{U} & =u(\bar{\theta}-\underline{q})-u(\underline{\theta}-\underline{q}) \\
& =u(\Delta \theta+\underline{\theta}-\underline{q})-u(\underline{\theta}-\underline{q})<u(\Delta \theta)
\end{aligned}
$$

from the concavity of $u(\cdot)$ and the fact that the optimal $\underline{q}$ in this regime defined by

$$
u^{\prime}(\underline{q})=u^{\prime}(\underline{\theta}-\underline{q})+\frac{\nu}{1-\nu}\left[u^{\prime}(\underline{\theta}-\underline{q})-u^{\prime}(\bar{\theta}-\underline{q})\right]
$$

is less than $\underline{\theta}$. Therefore this regime is impossible.

Another regime is obtained when the $\bar{\theta}$ 's participation constraint and the two enforce-

ment constraints are binding. Then $\underline{q}=\underline{\theta}$ and $\bar{q}=\frac{\bar{\theta}}{2}$. However, the principal's welfare obtained in this case, namely

$$
2 \nu u\left(\frac{\bar{\theta}}{2}\right)+(1-\nu) u(\underline{\theta})-\nu u(\Delta \theta)
$$

is less than the welfare obtained in regime 1 characterized in the text, namely

$$
2 \nu u\left(\frac{\bar{\theta}}{2}\right)+2(1-\nu) u\left(\frac{\theta}{2}\right)-\nu u(\Delta \theta),
$$

because of the concavity of $u(\cdot)$. 


\section{Appendix 2}

Consider now the case $\Delta \theta<\underline{\theta}$.

Regime 3 holds as long as the solution $\bar{q}_{3}$ of

$$
u^{\prime}\left(\bar{q}_{3}\right)=u^{\prime}\left(\underline{\theta}+\Delta \theta-\bar{q}_{3}\right)-\frac{(1-\nu)}{\nu}\left[u^{\prime}\left(\underline{\theta}-\bar{q}_{3}\right)-u^{\prime}\left(\underline{\theta}+\Delta \theta-\bar{q}_{3}\right)\right]
$$

is less than $\underline{\theta}$. So the definition of this regime depends on $\nu$.

For example, for $u(x)=x-\frac{x^{2}}{2}$, it requires $\Delta \theta \leq \nu \underline{\theta}$

$$
\begin{gathered}
W_{3}(\nu, \Delta \theta)=\nu\left(u\left(\bar{q}_{3}\right)+u\left(\bar{\theta}-\bar{q}_{3}\right)\right)+2(1-\nu) u\left(\frac{\underline{\theta}}{2}\right)-u(\Delta \theta) \\
+(1-\nu)\left(u\left(\bar{\theta}-\bar{q}_{3}\right)-u\left(\underline{\theta}-\bar{q}_{3}\right)\right) \\
\frac{\partial W_{3}}{\partial \nu}=u\left(\bar{q}_{3}\right)-u\left(\underline{\theta}-\bar{q}_{3}\right)-2 u\left(\frac{\underline{\theta}}{2}\right)<0
\end{gathered}
$$

from the concavity of $u(\cdot)$ for all $\bar{q}_{3} \leq \underline{\theta}$, i.e. both for Regimes 3 and 2

$$
\begin{aligned}
\frac{d W_{3}}{d \Delta \theta}=u^{\prime}\left(\bar{\theta}-\bar{q}_{3}\right)-u^{\prime}(\Delta \theta) & <0 \text { for } \bar{q}_{3}<\underline{\theta} \\
& =0 \text { for } \bar{q}_{3}=\underline{\theta} .
\end{aligned}
$$

The typical graph of welfare is presented in Figure 6.

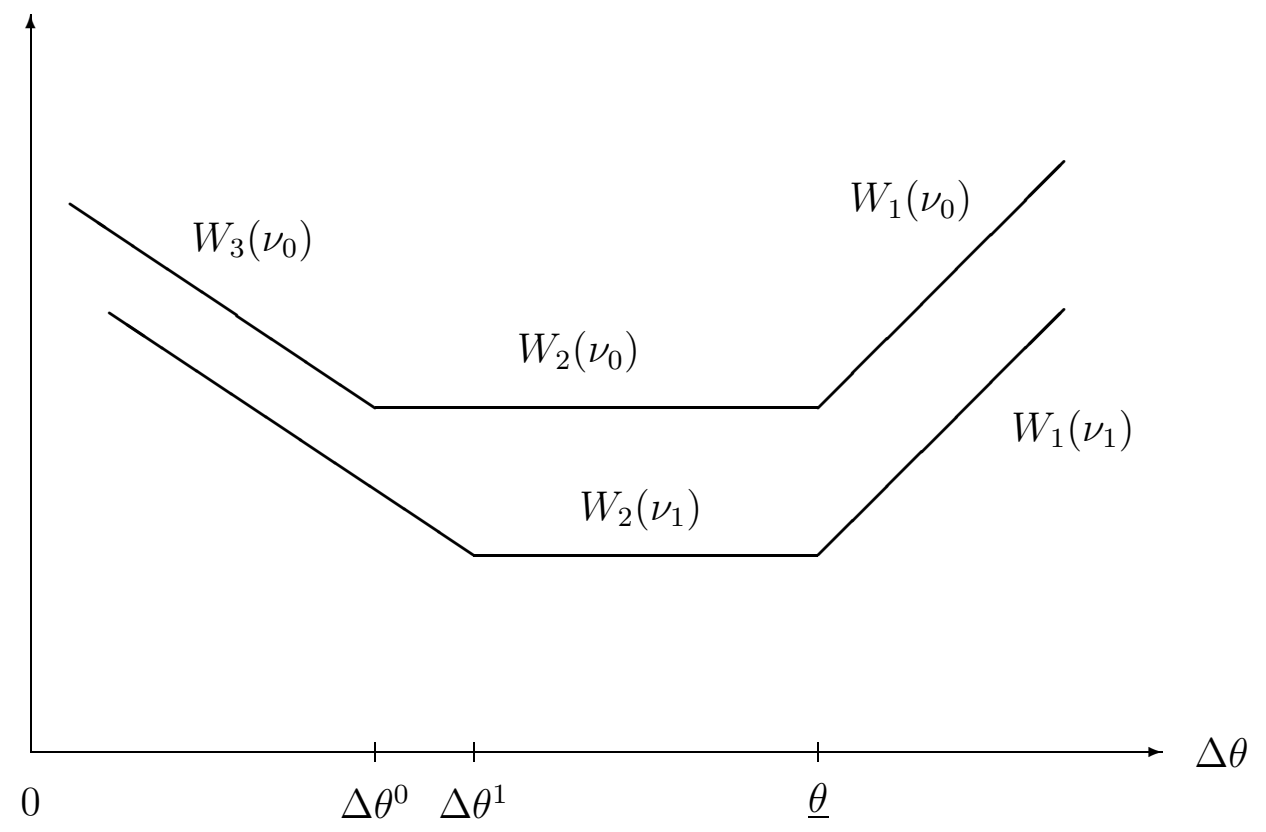

Figure 6 
Let $\Delta \theta^{i}$ be the value of $\Delta \theta$ at which the transition takes place between Regimes 3 and 2. Differentiating equation (1) at $\bar{q}_{3}=\underline{\theta}$ we obtain $\frac{d \Delta \theta}{d \nu}>0$. So $\Delta \theta^{1}>\Delta \theta^{0}$. For example for

$$
u(x)=x-\frac{x^{2}}{2}, \quad \Delta \theta^{1}=\nu_{1} \Delta \theta, \quad \Delta \theta^{0}=\nu_{0} \Delta \theta
$$

So, if we are in Regime 3 for $\nu=\nu_{0}$ we remain in Regime 3 for $\nu=\nu_{1}$.

The principal's problem is then

$$
\max \nu_{0}(u(\bar{q})-\bar{t})+\left(1-\nu_{0}\right)(u(\underline{q})-\underline{t})
$$

s.t.

$$
\begin{aligned}
& u(\underline{\theta}-\underline{q})+\underline{t} \geq u(\underline{\theta}-\bar{q})+\bar{t} \\
& u(\bar{\theta}-\bar{q})+\bar{t} \geq u(\Delta \theta) \\
& u(\underline{\theta}-\underline{q})+\underline{t} \geq u(\bar{\theta}-\bar{q})+\bar{t}-\frac{\psi}{\Delta \nu} .
\end{aligned}
$$

If

$$
u(\underline{\theta}-\bar{q})+\bar{t} \geq u(\bar{\theta}-\bar{q})+\bar{t}-\frac{\psi}{\Delta \nu}
$$

or

$$
u(\bar{\theta}-\bar{q})-u(\underline{\theta}-\bar{q}) \leq \frac{\psi}{\Delta \nu},
$$

i.e., for $\Delta \theta$ small enough, the solution defined by $\underline{q}_{3}=\frac{\theta}{2}$ and $\bar{q}_{3}$ defined by (1) for $\nu_{0}$ holds.

For $\Delta \theta \geq \Delta \theta_{0}$ such that

$$
u\left(\bar{\theta}, \bar{q}_{3}\right)-u\left(\underline{\theta}-\bar{q}_{3}\right)=\frac{\psi}{\Delta \theta_{0}}
$$

$\bar{q}_{3}$ is ajusted to $\hat{q}$ such that

$$
u(\bar{\theta}-\hat{q})-u(\underline{\theta}-\hat{q})=\frac{\psi}{\Delta \nu}
$$

to satisfy the moral hazard constraint. Then, the principal's welfare is

$$
\begin{aligned}
\tilde{W}_{3}\left(\nu_{0}, \Delta \theta\right)=\nu_{0}[ & u(\bar{\theta}-\hat{q})+u(\hat{q})]-u(\Delta \theta) \\
& +2\left(1-\nu_{0}\right) u\left(\frac{\hat{\theta}}{2}\right)+\left(1-\nu_{0}\right)(u(\bar{\theta}-\hat{q})-u(\underline{\theta}-\hat{q})) .
\end{aligned}
$$

The principal gives up discouraging effort when $\Delta \theta$ reaches $\Delta \theta_{1}$ such that

$$
\tilde{W}_{3}\left(\nu_{0}, \Delta \theta_{1}\right)=W_{3}\left(\nu_{1}, \Delta \theta_{1}\right) \text {. }
$$

Consider now $\Delta \theta$ such that we are in Regime 2 for $\nu_{0}$. The principal's program is

$$
\max \nu_{0}(u(\bar{q})-\bar{t})+\left(1-\nu_{0}\right)(u(\underline{q})-\underline{t})
$$




$$
\begin{aligned}
& u(\bar{\theta}-\bar{q})+\bar{t}=\Delta \theta \\
& u(\underline{\theta}-\underline{q})+\underline{t}=\max \left(0, u(\Delta \theta)-(u(\bar{\theta}-\bar{q})-u(\underline{\theta}-\bar{q})), u(\Delta \theta)-\frac{\psi}{\Delta \nu}\right) .
\end{aligned}
$$

When $u(\Delta \theta)<\frac{\psi}{\Delta \nu}$, the solution of Regime 2 discourages effort.

When $u(\Delta \theta)$ becomes larger than $\frac{\psi}{\Delta \nu}, \bar{q}$ is ajusted downward from $\underline{\theta}$ such a way that

$$
(u(\bar{\theta}-\bar{q})-u(\underline{\theta}-\bar{q}))=\frac{\psi}{\Delta \nu} .
$$

Then, depending on the values of parameters as $\Delta \theta$ increases further, the principal gives up discouraging effort and moves either to Regime 3 or Regime 2 and $\nu=\nu_{1}$. 


\section{Appendix 3}

Let us for simplicity increase the quality of enforcement $p$ exogenously. We then obtain the following profiles of expected welfare for the principal when we abstract from the cost of effort (see Figure 7).

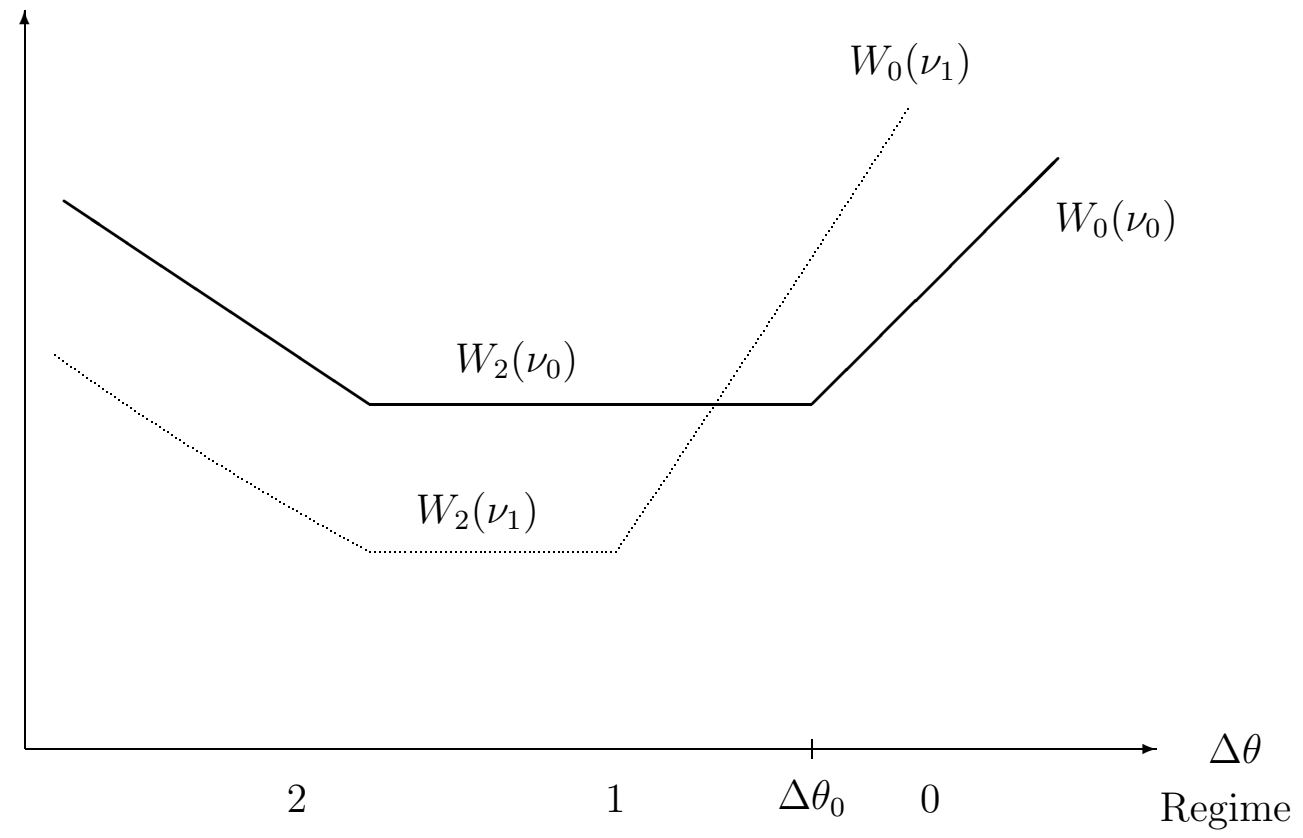

Figure 7

$\Delta \theta_{0}$ is determined by

$$
u\left(\Delta \theta_{0}-\frac{\underline{\theta}}{2}\right)-u\left(\frac{\underline{\theta}}{2}\right)=(1-p) u\left(\Delta \theta_{0}\right),
$$

i.e., independently of $\nu$.

Then, depending on the value of $\psi$ and $\Delta \theta$, the principal chooses or not to induce (in Regime 0) or discourage (in Regimes 1, 2, 3) effort. 


\section{Appendix 4: The Continuous Type Case}

Similar insights can be gained in the case where $\theta$ belongs to an interval $[\underline{\theta}, \bar{\theta}]$ and is distributed according to the distribution $F(\theta)$ with a positive density $f(\cdot)$. We also assume the hazard rate properties

$$
\frac{d}{d \theta} \frac{F(\theta)}{f(\theta)} \geq 0 \quad \text { and } \quad \frac{d}{d \theta} \frac{1-F(\theta)}{f(\theta)} \leq 0
$$

to avoid bunching of a classical type.

It is easily shown that with a renegation constraint of the type

$$
U(\theta) \geq(1-p) u(\theta)
$$

we have no countervailing incentives.

Proposition 4: With the renegotation constraint (1) the optimal contract entails downward distortion characterized by:

$$
u^{\prime}\left(q^{*}(\theta)\right)=u^{\prime}\left(\theta-q^{S B}(\theta)\right)-\frac{1-F(\theta)}{f(\theta)} u^{\prime \prime}\left(\theta-q^{S B}(\theta)\right)
$$

and a rent

$$
U=(1-p) u(\underline{\theta})+\int_{\underline{\theta}}^{\theta} u^{\prime}\left(\tau-q^{S B}(\tau)\right) d \tau .
$$

Renegation constraints simply oblige the principal to give up an additional rent (1 p) $u(\underline{\theta})$ to each type.

With the renegation constraint

$$
U(\theta) \geq u(\theta-\underline{\theta})
$$

one must distinguish two cases.

For $\Delta \theta$ small (more precisely for $\Delta \theta$ such that the solution $q(\theta)$ of the equation below is such that $q(\theta)<\underline{\theta}$ ) we have countervailing incentives:

Proposition 5: Under the renegotiation constraint (2), we have:

- For $\theta$ in $\left[\underline{\theta}, \theta_{0}\right]$, production $q_{3}(\theta)$ is upward distorted.

- For $\theta$ in $\left[\theta_{0}, \theta_{1}\right]$, there is bunching and $q_{2}(\theta)=\underline{\theta}$.

- For $\theta$ in $\left[\theta_{1}, \bar{\theta}\right]$, production $q_{1}(\theta)$ is downward distorted. 
Rents are

$$
\begin{aligned}
U(\theta) & =\int_{\underline{\theta}}^{\theta} u^{\prime}\left(\tau-q_{3}(\tau)\right) d \tau \text { for } \theta \text { in }\left[\underline{\theta}, \theta_{0}\right] \\
& =u(\theta-\underline{\theta}) \text { for } \theta \text { in }\left[\theta_{0}, \theta_{1}\right] \\
& =u(\bar{\theta})-\int_{\theta}^{\bar{\theta}} u^{\prime}(\tau-q(\tau)) d \tau \text { for } \theta \text { in }\left[\theta_{1}, \bar{\theta}\right] .
\end{aligned}
$$

The main difference between the two types of renegation constraints is as follows.

For (1), the marginal utility of the resource is lower for the agent when for the principal. The solution entails simply a bonus for the agent which is paid each time there is a discovery whatever its value.

For (2), the marginal utility of the resource is higher for the agent. Then, for discoveries of small variance, countervailing incentives prevail and lead to decrease the share of the resource left to the agent to increase his marginal utility for the good and decrease his rent.

For greater variance of discoveries, for which the marginal utility of the resource for the agent is higher and then lower than for the principal as $\theta$ increases, we have a complex solution sharing the features of the two cases above as $\theta$ increases with a bunching region in between.

Figure 8 describes the profile of quantities ${ }^{15}$

\footnotetext{
${ }^{15}$ Additional assumptions are needed to avoid bunching when $u^{\prime \prime}(\cdot)$ is not constant.
} 


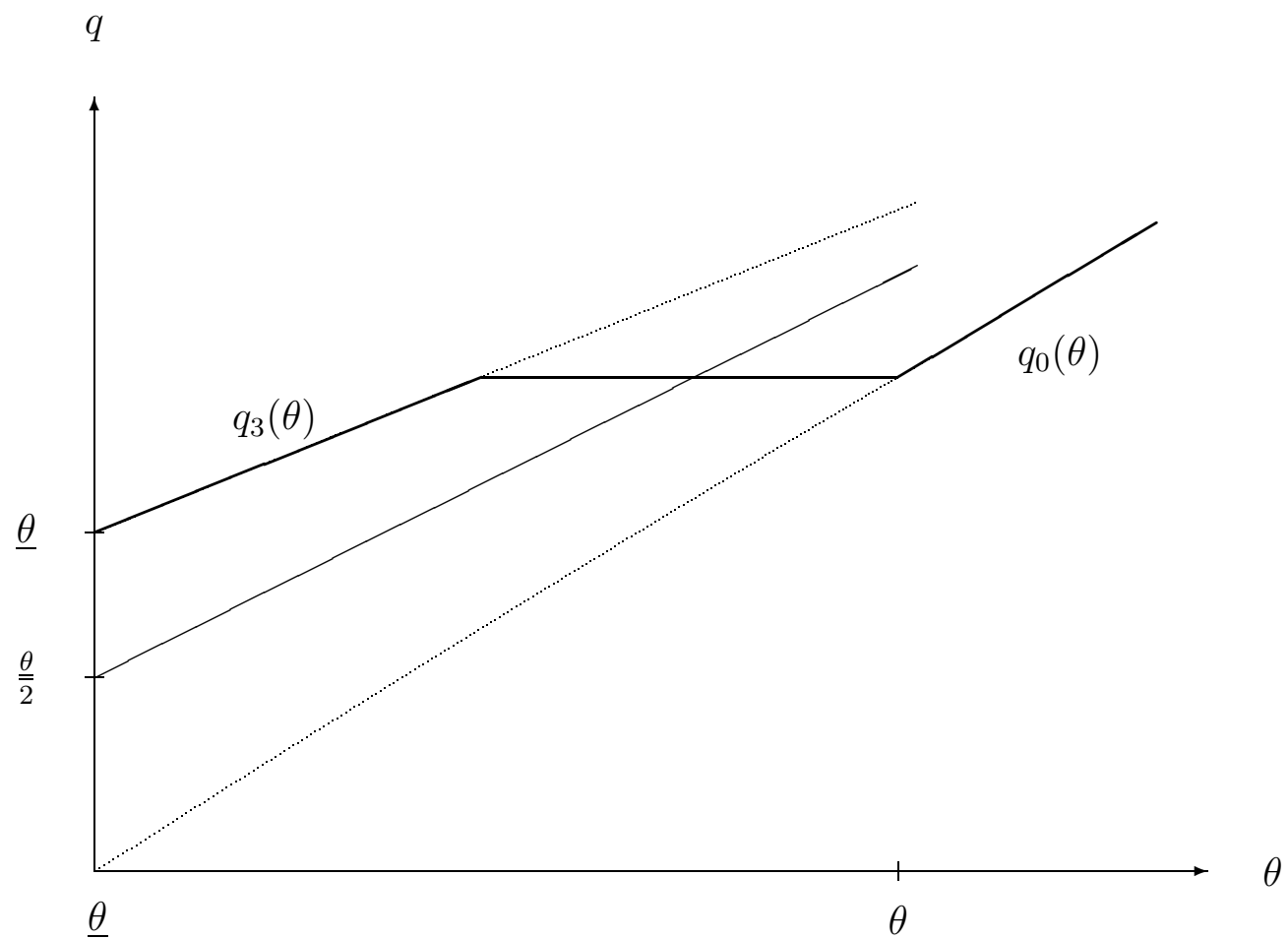

Figure 8

$$
\begin{aligned}
& u^{\prime}\left(q_{3}(\theta)\right)=u^{\prime}\left(\theta-q_{3}(\theta)\right)+\frac{F(\theta)}{f(\theta)} u^{\prime \prime}\left(\theta-q_{3}(\theta)\right) \\
& u^{\prime}\left(q_{0}(\theta)\right)=u^{\prime}\left(\theta-q_{0}(\theta)\right)-\frac{1-F(\theta)}{f(\theta)} u^{\prime \prime}\left(\theta-q_{0}(\theta)\right) .
\end{aligned}
$$




\section{References}

Bull J. and J. Watson (2001), "Evidence Disclosure and Verifiability", mimeo, University of California at San Diego.

Becker, G. (1968), "Crime and Punishment: An Economic Approach", Journal of Political Economy, 76, 169-217.

Becker, G. and G. Stigler (1974), "Law Enforcement, Malfeasance, and Compensation of Enforcers", Journal of Legal Studies, 3, 1-19.

Gibbard, A. (1973), "Manipulation of Voting Schemes: A General Result", Econometrica, 41, 587-601.

Green, J. and J.J. Laffont (1977), "Characterization of Strongly Individually Incentive Compatible Mechanisms for the Revelation of Preferences for Public Goods", Econometrica, 45, 427-438.

Green, J. and J.J. Laffont (1986), "Partially Verifiable Information and Mechanism Design", Review of Economic Studies, 53, 447-456.

Hurvicz, L. E. Maskin and A. Postlewaite (1982), "Feasible Implementation of Social Choice Correspondences by Nash Equilibria", mimeo.

Jullien, B. (2000), "Participation Constraint in Adverse Selection Models", Journal of Economic Theory, 93, 1-47.

Laffont, J.J. (2001), "Enforcement, Regulation and Development", to appear in Journal of African Economics.

Laffont, J.J. and D. Martimort (2002), The Theory of Incentives, Princeton University Press, Princeton.

Laffont, J.J. and J. Tirole (1990), "Bypass and Creamskimming", American Economic Review, 80, 1042-1061.

Lewis, T. and D. Sappington (1989), "Countervailing Incentives in Agency Problems", Journal of Economic Theory, 49, 294-313.

Maggi, G. and A. Rodriguez-Clare (1995), "On Countervailing Incentives", Journal of Economic Theory, 66, 238-263.

Myerson, R. (1979), "Incentive Compatibility and Bargaining", Econometrica, 47, 61-73.

Postlewaite, A. (1979), "Manipulation via Endowments", The Review of Economic Studies, 66, 255-262.

Stigler, G. (1970), "Optimum Enforcement of Laws", Journal of Political Economy, 78, 526-536. 